\section{$\underset{\substack{\text { hommes } \\ \text { \& migrations }}}{ }$}

\section{Hommes \& migrations}

Revue française de référence sur les dynamiques

migratoires

\section{$1279 \mid 2009$}

L'Afrique en mouvement

\title{
Migration interne et acculturation
}

Le cas des migrants bassari à Tambacounda

\section{Babacar N'Dong}

\section{(2) OpenEdition}

1 Journals

\section{Édition électronique}

URL : http://journals.openedition.org/hommesmigrations/299

DOI : 10.4000/hommesmigrations.299

ISSN : 2262-3353

Éditeur

Musée national de l'histoire de l'immigration

\section{Édition imprimée}

Date de publication : 1 mai 2009

Pagination : 52-64

ISSN : 1142-852X

\section{Référence électronique}

Babacar N'Dong, « Migration interne et acculturation », Hommes \& migrations [En ligne], 1279 | 2009,

mis en ligne le 29 mai 2013, consulté le 03 mai 2019. URL : http://journals.openedition.org/

hommesmigrations/299; DOI : 10.4000/hommesmigrations.299 


\section{Migration inteme et d'acculturation}

Le cas des migrants bassari à Tambacounda

Par Babacar N'Dong, anthropologue, université Pierre Mendès France, Grenoble

En Afrique, les migrations intemes, qui ont conduit au peuplement du continent, se poursuivent. La recherche de terres fertiles ou d'un travail rémunéré, la fuite devant un ennemi ou une catastrophe naturelle, continuent de pousser les migrants à partir. Dans la rencontre avec

d'autres cultures se joue la préservation de leurs coutumes. Longtemps resté isolé dans une zone de collines et de forêts denses, le peuple bassari fait aujourd'hui face à un enjeu majeur du processus de migration inteme : son acculturation. 


\section{Qui sont les Bassari?}

Les Bassari et leurs voisins bedik, coniagui, badiaranké et boïn appartiennent au groupe ethno-linguistique tenda ${ }^{(1)}$. Ils se dénomment eux même Beliyan, c'est à dire "fils de la terre latéritique". Estimés environ à 36130 individus, ils sont répartis de part et d'autre de la frontière sénégalo-guinéenne, sur les contreforts de la montagne du Fouta Djalon entre la frontière des deux Guinée et de la nouvelle région de Kédougou. Le pays bassari est situé à environ 400 kilomètres de la côte atlantique et à 800 kilomètres de Dakar par la route. Les villages bassari se répartissent sur un territoire de 60 kilomètres de long et 15 kilomètres de large.

Initialement, ils formaient un peuple de chasseurs-cueilleurs. Avec l'accroissement de la population et des champs, le gibier est devenu rare. Certains animaux comme l'éléphant ont disparu avec le braconnage, dans ce qui est devenu le parc national de Niokolo Badiar. L'interdiction de chasser dans le parc pose problème aux Bassari et à leurs voisins tenda, dans la mesure où elle les oblige à modifier une bonne partie de leurs habitudes alimentaires. La viande de chasse issue du braconnage est progressivement remplacée par celle des animaux domestiques.

La population se partage entre six à huit clans matrilinéaires selon les villages. L'appartenance à une lignée se transmet par la mère. L'organisation sociale est essentiellement basée sur un système de classe d'âge. De la naissance à la mort, on appartient à une classe d'âge. Chacune d'elles comporte des obligations, des prestations et des cérémonies. Le rituel de passage d'une classe d'âge à l'autre a lieu tous les six ans.

L'initiation est l'une des plus importantes phases de la vie des adolescents dans la société bassari. Elle a lieu à l'âge de 14-15 ans. Elle met fin, pour l'adolescent, à sa condition d' "homme marginal".

\section{Une communauté fortement structurée}

Jusqu'à la fin du XIX siècle, les Bassari vivaient en "économie de ponction" "(2). La redistribution du surplus était la règle, le troc permettait de pallier le déficit alimentaire. L'organisation économique était de type communautaire. Les classes d'âge juvéniles participaient aux travaux agricoles des familles, gagnant ainsi de quoi fournir à leurs aînés des compensations en bière. L'usage de l'argent n'a fait son apparition qu'à partir des années trente ${ }^{(3)}$. Les Bassari ont longtemps vécu dans un grand isolement, repliés dans les zones de collines. Une façon d'échapper à la pression des pasteurs peuls islamisés venus du Fouta Djallon voisin, qui s'accaparaient leurs 
terres, enlevaient leurs femmes, réduisaient leurs enfants en esclavage et tentaient de les convertir. Selon Monique Gessain, vers les années dix-neuf cent, la population bassari était stationnaire, et leur société presque autarcique. Ils étaient peu nombreux, ne se mariaient qu'entre eux et n'entretenaient que des relations de commerce avec leurs voisins les plus proches. Ils ne dépendaient de l'extérieur

\section{A partir des années trente, les Bassari ont commencé à accompagner \\ leurs voisins coniagui dans les plantations de sisal de Wassadou, en Gambie ou encore en Guinée voisine.} que pour leurs besoins en sel.

Sur le plan religieux, la société bassari se caractérise par la croyance à une interpénétration entre le monde visible et le monde invisible peuplé par les génies et les esprits des ancêtres morts (biyil). Cette croyance leur a permis de vivre en harmonie avec la nature qui les entoure, d'en tirer profit sans pour autant lui porter de graves préjudices. Ce respect de l'écosystème était facilité par l'occupation de l'espace. Les familles vivaient éloignées les unes des autres. Entre 2 et 5 kilomètres pouvaient séparer deux concessions.

À cette époque, les fêtes et loisirs occupaient une très grande place dans le calendrier annuel des Bassari. Les jeunes n'allaient pas à l'école, ce qui leur permettait de suivre tous les rites de passage qui jalonnaient leur vie d'adulte. La société bassari a donné naissance à plusieurs types de masques, et chaque masque possède son calendrier de sorties, ses rituels d'accompagnement et une fonction spécifique. Les matériaux utilisés (bois, fibres, feuilles) sont différents selon qu'il s'agit d'un masque d'initiation dit sacré ou d'un masque de danse. Cette richesse culturelle a très vite attiré des chercheurs mais aussi des touristes. Ce qui a contribué à l'ouverture progressive du pays bassari.

\section{Une ouverture au monde récente}

À partir des années trente, les Bassari ont commencé à accompagner leurs voisins coniagui dans les plantations de sisal de Wassadou, en Gambie, ou encore en Guinée voisine. À cette époque, certains (des militaires en particulier) commencent à se rendre dans les grandes villes du Sénégal. Dans le même temps, les premières missions chrétiennes arrivent chez les Bassari, accompagnées des premières écoles. Ce contact avec l'extérieur leur a permis de découvrir de nouveaux produits, ce qui entraîne progressivement leur passage du statut de chasseurs-cueilleurs à celui d'agriculteurs. 
Il faut attendre 1960, c'est-à-dire la période qui suit les Indépendances, pour observer une réelle ouverture du pays bassari au monde extérieur. Les Bassari se trouvent alors répartis entre la Guinée et le Sénégal. Sur le plan politique, les administrations sénégalaises et guinéennes relaient l'administration française. L'interdiction de certaines coutumes bassari en Guinée va les détourner de ce pays et les orienter vers les grandes villes du Sénégal.

Cette émigration concerne exclusivement les hommes. Ils partent en groupe à la saison sèche dans les grandes villes comme Kédougou et Tambacounda. Rares sont ceux qui vont jusqu'à la capitale. Ils reviennent tous dès l'approche de l'hivernage pour assister aux différentes coutumes, telle que l'initiation des jeunes garçons, mais aussi participer aux travaux champêtres. Ces migrations sont surtout motivées par la recherche de l'argent afin de payer l'impôt ou de compléter la dot pour se marier. En ville, les garçons sont employés comme gardiens ou jardiniers. De retour au village, ils jouissent d'un grand prestige social auprès de la population, surtout féminine, ce qui pousse beaucoup de jeunes à émigrer l'année suivante.

\section{Le territoire bassari à l'épreuve des flux économique et touristique}

L'organisation économique, qui jusqu'ici était fondée sur le troc, va se monétariser. C'est la naissance des marchés hebdomadaires, comme celui d'Oubadji en $1961^{(4)}$, et l'apparition d'une diversité de produits en pays bassari. C'est aussi l'arrivée de certaines variétés comme l'arachide et les légumineuses et plus tard le coton, en 1974, avec la SODEFITEX (Société d'exploitation des fibres textiles). Les missions catholiques s'installent dans presque tous les villages tenda.

En 1994, un événement important se produit pour le désenclavement du Sénégal oriental. Une route nationale est ouverte entre Tambacounda et Kédougou, chef-lieu du département, où vit la majorité des Bassari. Il est désormais possible d'atteindre le pays bassari depuis Dakar en un peu plus d'une journée de route. Cela accentue les flux touristiques qui, sans être massifs, deviennent tout de même assez conséquents pour que quelques habitants du pays ouvrent des campements d'accueil avec des cases en latérite, typiques de l'architecture locale, organisent la vente d'objets artisanaux et fondent des troupes de danseurs et de musiciens qui se produisent pour les visiteurs en s'inspirant de leurs cérémonies traditionnelles. Le désenclavement de la région, tout relatif qu'il soit, a aussi facilité l'exode rural.

Depuis quelques années, on observe l'installation de véritables communautés bassari à Kédougou et à Tambacounda, chef-lieu de la région. Dans cette dernière ville, les 
Bassari se sont regroupés dans des quartiers en périphérie urbaine sans eau ni électricité. On y trouve des familles entières vivant des différentes activités que l'on peut pratiquer dans une agglomération de cette importance.

\section{Présenver sa culture en milieu urbain}

Les Bassari installés à Tambacounda ont conservé certains aspects essentiels de leurs traditions. On observe chez eux la perpétuation des fêtes avec les sorties de masques et l'attachement aux divers rituels qui rythment la vie dans le milieu traditionnel. Cependant, ce maintien des pratiques culturelles et religieuses, constitutives de l'identité de leur peuple, se fait au prix d'une stratégie de limitation des contacts avec les autres populations présentes en ville.

Les Bassari se déplacent chaque fois que leur quartier de résidence fait l'objet d'aménagement et en particulier chaque fois qu'il y a électrification. Cet intérêt pour l'obscurité pose question. Il n'est pas sans évoquer les mythes originels qui parlent d'un habitat troglodyte ni les épisodes historiques plus récents où, menacés par leurs voisins peuls, ils se réfugiaient dans les grottes. Les quartiers obscurs et mal équipés apparaissent comme un lieu protecteur où le groupe peut espérer réduire les contacts avec les autres citadins. La lumière apparaît aussi comme un danger pour perpétuer le secret des masques. Car au cours des danses nocturnes, le visage des porteurs de masque ne doit pas être révélé.

Il y a en effet un risque objectif de déstructuration de la société bassari, du fait de son originalité par rapport aux groupes de population présents en ville. Les Bassari forment l'une des rares sociétés à avoir conservé un mode de filiation de type matrilinéaire dans un pays où la plupart des sociétés sont patrilinéaires et où le code civil, inspiré du Code Napoléon, considère que les enfants doivent porter le nom de leur père. L'exogamie est donc un danger pour le maintien de ce mode de filiation, tout comme le contact que les enfants entretiennent avec des enfants appartenant à d'autres groupes, en particulier à travers l'école.

\section{Une vie à la périphérie}

Avec l'augmentation très rapide de la population urbaine, les Bassari ont tendance à se replier dans des quartiers lointains. Il faut dire qu'à Tambacounda ils ne sont pas les premiers à abandonner leurs maisons dès que leur quartier commence à s'urbaniser. Ceux de Kédougou avaient déjà abandonné leur quartier au moment 
de l'électrification et de l'adduction d'eau. L'urbanisation est synonyme de lotissement, ce qui attire beaucoup de populations et fait augmenter le prix du loyer, mais, surtout, le prix des terrains avec la spéculation foncière. La dernière opération de lotissement devait coûter 50000 francs CFA à chaque propriétaire pour qu'il puisse

\section{Dans le contexte du Sénégal contemporain, les Bassari ont l'image d'une "population sauvage" qui, il y a quelques décennies, vivait encore presque nue dans les zones rurales.} disposer officiellement de sa maison. Le salaire moyen des Bassari, compris entre 15 et 25000 francs par mois, explique leur éloignement des quartiers urbanisés.

Avec le lotissement du quartier Médina Coura en 1972, beaucoup ont vendu ou simplement préféré laisser sur place leur maison pour aller s'installer un peu plus loin. Ce même phénomène s'est reproduit en 1978 et en 1994 avec le lotissement du quartier Gourel Diadji qui poussa les Bassari à aller s'installer dans un nouveau quartier qu'ils baptisèrent Pona, nom du premier occupant. En s'installant à la périphérie de la ville, ils peuvent continuer à perpétuer certains aspects de leurs coutumes comme les sorties de masques, qui sont souvent l'occasion de fêtes et danses qui peuvent durer plusieurs jours. À ces deux aspects, il faudrait ajouter la pratique de l'agriculture et de l'élevage, la chasse et enfin la vente de la bière qui est l'une des activités principales des femmes.

\section{Du repli sur soi au spectacle culturel}

Cette population, installée à la marge de la ville, vit malgré tout un processus d'acculturation qui, à long terme, risque de transformer profondément son organisation traditionnelle. Les diverses études sur les communautés immigrées, en particulier celles qui se sont déroulées aux États-Unis ${ }^{(5)}$ ont toujours fait ressortir que, dans un premier temps, on observe chez les populations récemment installées en ville une tendance au repli. Les immigrés récents tendent toujours à créer une "enclave ethnique", avec un souci de conservation des traditions qui peut paraître parfois plus affirmé que ce que l'on peut observer dans le milieu d'origine. Les communautés indigènes ont pour point commun de ne pas (encore) être diluées dans le monde qui les entoure.

Une abondance de formes sociales subsiste à l'uniformisation du monde entraînée par la globalisation du marché et par la mondialisation des rapports sociaux. Dans le contexte du Sénégal contemporain, les Bassari ont l'image d'une "popula- 
tion sauvage" qui, il y a quelques décennies, vivait encore presque nue dans les zones rurales. Les fêtes accompagnées de libations excessives et la permissivité sexuelle qui caractérise les rapports entre hommes et femmes ajoutent à l'image négative qu'ils ont encore auprès de leurs voisins.

En même temps, le récent développement du tourisme dans la région et les politiques de valorisation des cultures locales, à travers les manifestations organisées par les autorités politiques, ont contribué à mettre en avant certains de leurs rites et certaines de leurs fêtes. Mais cette valorisation de leur culture à travers le spectacle culturel présente le risque de faire perdre le sens de ces diverses manifestations, en particulier aux jeunes qui ne connaissent plus très bien le milieu traditionnel. Aujourd'hui, avec l'installation de plus en plus de migrants de longue durée à Tambacounda et l'augmentation du nombre de migrants saisonniers, les contacts entre ville et village se font de plus en plus fréquents. Les enfants sont de plus en plus nombreux à être scolarisés. Ils passent plus de temps à l'école, ce qui raccourcit la durée de l'initiation, qui était d'un an. Beaucoup de parents estiment aujourd'hui que la réussite de leurs enfants passe par l'école et non par l'accomplissement de l'ensemble des rites appelés A-tuwin.

\section{Le christianisme, levier de l’acculturation}

Souvent, les migrants sont dominés par les autochtones, qui leur font perdre leur langue et leurs coutumes. Ce "nouveau peuple (6)", qui se façonne au fil des générations, tend à oublier son origine en s'appropriant la mémoire collective du groupe d'accueil. Si l'on définit l'acculturation comme un processus de transformation culturelle qui se traduit par l'acquisition de nouvelles normes, valeurs, rituels et coutumes, n'impliquant pas la disparition totale des anciens repères, alors on peut dire que la population bassari installée à Tambacounda vit actuellement un processus de ce type. La conversion au christianisme constitue le vecteur principal de cette acculturation.

La présence des missions chrétiennes, autant catholiques que protestantes, en milieu traditionnel bassari est une réalité ancienne. Mais nous avons pu remarquer que le nombre de chrétiens parmi les émigrés partis de chez eux depuis longtemps est assez élevé, comparé à la proportion des chrétiens, bien plus faible, parmi les Bassari restés vivre au village. Dans ce milieu traditionnel, les résistances culturelles à la pénétration du christianisme sont plus fortes qu'en ville. En 2005, dans le village d'Ebarack, les missionnaires avaient béni les futurs initiés, avant le combat contre les masques. Les personnes âgées avaient manifesté 
leur mécontentement devant cette intrusion dans une cérémonie traditionnelle, estimant que cela constituait un danger pour la culture bassari.

Les conversions au christianisme dans une ville comme Tambacounda, très majoritairement musulmane, constituent un signe évident d'acculturation. Le succès du christianisme chez les Bassari devenus citadins s'explique par la possibilité de retrouver dans les rites chrétiens des éléments existant au niveau de la religion traditionnelle.

C'est ce que nous révèle un homme d'Église interviewé au cours de nos enquêtes. Selon lui, il est possible de trouver des correspondances entre les sacrements de l'Église et les différents rites bassari :

"Grâce à la traduction et à la connaissance de la culture bassari, nous essayons de montrer les liens entre les rites de passage tant dans la religion traditionnelle bassari que dans le catholicisme. Nous faisons le rapprochement à travers les sacrements que sont le baptême, la communion et la confirmation. A chaque sacrement, on fait correspondre une classe d'âge ou un rite spécifique.

Le baptême correspond à linitiation, la communion à la classe des o-palug et la Confirmation à celle des o-dyar.".

Nous leur expliquons que de la même façon que linitiation est fondamentale chez les Bassari, le baptême est aussi fondamental chez les catholiques. Nous leur disons aussi que de la même façon que les o-dyar sont les piliers de la société bassari grâce à leur rôle d'interface entre les jeunes et les vieux, ils témoignent de leur bonne foi à travers la confirmation. Nous essayons donc de faire passer le message religieux par le biais des traditions ${ }^{(8)}$."

Cette tactique de conversion par la recherche des correspondances entre les rituels a été observée à plusieurs reprises en Amérique latine ${ }^{(9)}$. Le fait de devenir catholiques en ville permet aussi aux Bassari de former une communauté pouvant disposer d'une organisation mieux adaptée au milieu urbain.

\section{Une religion à usage social}

Après le brevet ou le baccalauréat, plusieurs jeunes sont recueillis dans les centres d'hébergement que les missions ont ouverts en ville. Pendant leur séjour dans ces centres d'hébergement, ils sont amenés tous les jours, matin et soir, à faire la prière et à apprendre les chants religieux. Ils sont regroupés en différentes chorales et, deux fois par semaine, ils se retrouvent pour les répétitions. Il existe aujourd'hui d'importants noyaux d'émigrés bassari autour de ces missions. Cela peut expliquer pourquoi certains d'entre eux finissent par choisir de s'investir dans l'Église, pour après retourner prêcher auprès de leurs compatriotes. 
L'avantage aussi pour l'Église de compter des Bassari en son sein est lié à la langue. Il est certain qu'il est plus facile à un pasteur ou à un prêtre bassari d'officier auprès des siens qu'à une personne appartenant à un autre groupe ethnique. À Tambacounda, nous comptons parmi les migrants de longue durée des prêtres, des pasteurs, des catéchistes et des séminaristes pour les plus jeunes. Une chapelle a même été édifiée dans le quartier Gourel Djiadji, où beaucoup résident, près de la maison du chef des Bassari. Les "hommes d'Église" jouent un rôle très important auprès de la communauté bassari. Tout en incitant les gens à abandonner les pratiques qui leur paraissent incompatibles avec le christianisme, ils les amènent à maintenir le lien communautaire, en conservant une originalité religieuse de type nouveau, dans un environnement essentiellement musulman.

"En tant qu'homme d'Église bassari, jessaye d'apporter ma contribution en discutant avec la population. Je leur explique que dans la religion traditionnelle bassari, il y a des choses qu'il faut abandonner.

Jadis très respectueux de la nature, les Bassari s'adonnent aujourd'hui à la recherche effrénée de terres. L'étendue de la forêt sacrée, réservée aux biyil, diminue progressivement. Cependant, il y a des choses positives aussi. C'est grâce à la religion catholique que le sacrifice humain a été abandonné. Nous luttons aussi contre l'adoration et la perpétuation du culte des ancêtres. Tous les sacrifices qui sont fait sur les autels sont interdits par la religion catholique. Nous leur expliquons que le Christ s'est sacrifié pour tout le monde. Par contre en ce qui concerne les chants et danses bassari, nous les intégrons dans les prières. Nous estimons que chacun peut vivre et pratiquer la religion tout en gardant ses coutumes. Autrement dit, on peut être catholique et bassari à la fois."

\section{Transformation ou disparition ?}

Quoi qu'il en soit, cette évolution religieuse coïncide avec une transformation du rapport de la société bassari avec son environnement traditionnel. Dans les zones d'origine, les cultures de rentes ont pris la place de la chasse et de la cueillette. Les terres arables diminuent, on raccourcit la période de jachère. Jadis très respectueux de la nature, les Bassari s'adonnent aujourd'hui à la recherche effrénée de terres. L'étendue de la forêt sacrée, réservée aux biyil, diminue progressivement. D'autres transformations significatives s'observent au niveau de l'habitat et du rapport à la nature. De la hutte, ils sont passés à la case ronde en blocs de pierre. Aujourd'hui, de plus en plus de gens ont adopté la case carrée, plus facile à aménager. La transformation de l'habitat traduit aussi une évolution des mentalités et 
des comportements. Autrement dit, si la case ronde représente la case collective, avec un sens profond de la solidarité de groupe, la case carrée offre, quant à elle, plus de liberté et d'indépendance par rapport au reste du groupe. Toutefois, cette indépendance n'est pas synonyme d'individualisme, car, même en ville, la notion de collectivité reste importante chez les Bassari.

En ville, ils découvrent également la médecine moderne. Ainsi, les Bassari sont de plus en plus nombreux à se rendre dans les hôpitaux et les dispensaires. Le recours aux vertus des plantes médicinales se fait de moins en moins. Les jeunes, y compris ceux qui sont nés au village ignorent une bonne partie de la pharmacopée traditionnelle. À cela s'ajoute le fait que la majeure partie de ces plantes n'est pas disponible en ville. Pourtant, l'histoire de la société bassari montre que celle-ci n'est pas figée, ni recroquevillée sur elle-même. D'ailleurs, dès 1965, Robert Gessain ${ }^{(10)}$ notait que l'étude des traditions n'est pas l'étude d'un passé mort : elle consiste à mettre à jour des changements. Toutes les sociétés ont toujours changé, dans leurs techniques, leurs structures, leurs croyances, et elles l'ont fait à des rythmes plus ou moins rapides, qui affectent diversement les différents niveaux culturels...

\section{La difficile résistance de l’ordre coutumier}

À travers son installation en ville, la communauté bassari apprend à cohabiter avec les autres groupes socio-ethniques et à exposer sa dynamique sociale à une modernité de plus en plus envahissante. Comparée aux autres migrations qui existent en Afrique, la migration des Bassari n'a rien d'exceptionnel. Mais dans leur cas, c'est une migration qui est en train de se dérouler, et donc observable. À cela s'ajoute le fait que les structures locales n'ont pas encore été abandonnées. Autrement dit, bien que la migration ait entrâné une dynamique de changement, autant au village que dans la communauté installée en ville, elle n’a pas encore provoqué de ruptures profondes et irréversibles avec la tradition. Mais on peut se demander si celle-ci résistera à l'épreuve du temps et aux inégalités que produit la vie en ville. L'augmentation très rapide de la population bassari vivant en ville $(2,7 \%$ de la population urbaine en 2006, contre 700 Bassari installés à Tambacounda, dans 130 maisons, en 1977, selon Nolan) montre que le centre de leur vie communautaire sera de moins en moins le milieu rural dans lequel se sont forgées leurs coutumes.

Aujourd'hui, beaucoup de jeunes naissent et grandissent en milieu urbain, ignorant une bonne partie des rites de passage. Seule l'initiation, qui est le rite le plus important, est toujours observée. Si les autres coutumes n'ont pas disparu, c'est qu'elles n'ont tout simplement jamais existé en ville. À Tambacounda, il n'y a 
jamais eu de case collective, de classes d'âge au sens traditionnel du terme, mais des associations. Sur le plan de l'organisation politique, il n'existe pas non plus à Tambacounda de collège d'o-nema $a^{(11)}$ responsable de la coutume.

On note aussi une "féminisation ${ }^{(12)}$ " de la communauté immigrée. Paradoxalement, si les femmes trouvent l'avantage d'échapper aux coutumes contraignantes, elles rencontrent souvent des difficultés liées à la solitude mais aussi à la perte de leur statut traditionnel. Contrairement au village, la matrilinéarité est moins observée en ville. L'influence des autres groupes ethniques et de l'administration sénégalaise pousse les gens à donner à leurs enfants le nom du père et non plus celui de la mère, ce qui porte atteinte au statut des femmes et induit des perturbations dans le jeu complexe des échanges matrimoniaux qui interdisent d'épouser une personne portant le même nom clanique.

\section{Les Bassari et la mondialisation : l'heure des choix}

Ainsi, les Bassari sont à un tournant décisif de leur histoire, autant sur le plan économique et social que culturel. Les possibilités qu'offre le séjour en ville sont nombreuses. En plus de pouvoir gagner un peu plus d'argent, il fait naître chez les jeunes des désirs nouveaux. L'autorité du pouvoir local est de plus en plus contestée. Certains commencent à trouver le cycle des coutumes a-tyuwin assez contraignant. Un certain nombre de personnes émigrent vers la ville pour échapper à ces coutumes. À cela s'ajoutent les changements apportés par la scolarisation, le tourisme, les nouvelles religions, etc. Tous ces facteurs sont annonciateurs de grands changements dans un futur proche et les Bassari ne s'y opposeront pas, n'en ayant ni les moyens, ni l'envie. Nous sommes à l'heure de la mondialisation et les Bassari n'en sont pas en marge. La question cruciale qui se pose aux Bassari et à toutes les ethnies dites minoritaires ou autochtones est de savoir comment entrer dans la mondialisation. Il n'y a que deux façons. La première conduit à l'aliénation, à la disparition de la culture bassari, mais aussi à long terme de toutes les cultures minoritaires sous l'influence des autres cultures. On serait dans un monde monochrome, homogène, uniforme. La seconde se fait dans le respect de la diversité culturelle, cette richesse incommensurable qui donne goût et sens à notre vie. Elle constitue un patrimoine commun de l'humanité qui doit à tout prix être préservé. C'est à ce titre que nous pensons que la préservation de la culture bassari relève à la fois des Bassari eux-même, de l'État sénégalais, mais aussi des instances internationales comme l'Unesco. Autrement dit, l'exception culturelle doit être considérée en droit international et 
bénéficier d'un traitement particulier. En ce sens, l'adoption par l'Unesco en octobre 2005 de la "Convention sur la protection et la promotion de la diversité des expressions culturelle" est un grand pas en avant.

Certes, la modernité a fait irruption dans la vie des Bassari, certes beaucoup ont aujourd'hui embrassé les religions importées, se sont installés en ville, mais dans leurs pratiques quotidiennes, les Beliyan continuent toujours de perpétuer le cycle initiatique qui fait de chaque adolescent un homme accompli. Nous croyons à la forte volonté du peuple bassari de survivre. Qu'il puisse, à l'image de son totem le caméléon, s'adapter aux influences extérieures tout en gardant ses particularités.

\section{Notes}

1. Le groupe tenda est constitué de plusieurs ethnies dont les Bassari, les Bedik, les Coniagui, les Boins.

Toutes ces ethnies vivent sur le même territoire situé au sud-ouest de la boucle formée par la Haute-Gambie au Sénégal. Les Tenda se caractérisent par la dimension de leurs communautés qui sont relativement petites, mais aussi par leurs coutumes qui sont restées vivaces.

2. Jean Girard, Les Bassari du Sénégal fils du caméléon: dynamique d'une culture troglodytique, Paris,

l'Harmattan, 2000, p. 10.

3. Monique Gessain, Les Migrations des Coniagui et des Bassari. Paris, Société des africanistes, 1967.

4. Alexandre Albenque, Les marchés hebdomadaires de la région de Kédougou-Sénégal oriental, in Bull. de l'Ifan,

T. XXXII, Série. B, $\mathrm{n}^{\circ} 1,1970$, p. 560.

5. En particulier, les travaux de H.Gans sur les villages urbains ou ceux de Louis Wirth sur le ghetto.

6. Sophie Le Callennec, "Les migrations africaines" in Ailleurs, l'herbe est plus verte : histoire des migrations dans

le monde. Collection Panoramiques, 1996, p. 85.

7. Cette classe regroupe les jeunes garçons de 21 à 27 ans qui sont les plus forts et les plus robustes du groupe.

Ils animent les danses pendant les festivités d'initiation.

8. Les extraits d'entretiens mentionnés dans cet article proviennent de la thèse de doctorat que nous avons soutenu en juillet 2008 à l'université de Grenoble 2 : Babacar N'Dong "La communauté bassari de Tambacounda, une société traditionnelle en milieu urbain", sous la direction de J. Barou.

9. Voir en particulier les travaux de Roger Bastide, dont "Les religions africaines au Brésil", Puf, 1995.

10. Robert Gessain, in Monique Gessain, Bassari : Guinée et Sénégal, op. cit., p. 56.

11. Les o-nema sont recrutés parmi les classes d'âge supérieures à partir des o-dyar (cette classe regroupe les jeunes garçons de 21 à 27 ans qui sont les plus forts et les plus robustes du groupe. Ils animent les danses pendant les festivités d'initiation). Chaque classe d'âge est représentée par deux o-nema. La sélection tient compte de la capacité de l'individu à diriger les membres de sa classe d'âge, de ses pouvoirs surnaturels, de son calme et de sa discrétion.

12. Abdou Salam Fall et Rokhaya Cissé, Migrations internationales et pauvreté en Afrique de l'Ouest, Ifan 2007.

\section{Bibliographie}

- Alexandre Albenque, "Les marchés hebdomadaires de la région de Kédougou-Sénégal Oriental", in Bull. de l'Ifan, t. XXXII, Série. B, nº1, 1970.

- Jacques Barou, La planète des migrants. Circulation migratoires et construction de diasporas à l'aube du XXIe siècle, Pug, 2007.

- Monique Gessain, Bassari : Guinée et Sénégal, 1927- 2002, Paris, Sépia, 2003.

- Monique Gessain, Les migrations des Coniagui et Bassari, Paris, Société des africanistes, 1967.

- Jean Girard, Les Bassari du Sénégal : fils du caméléon. Dynamique d'une culture troglodytique, Paris, l'Harmattan, 2000.

- Sophie Le Callennec, "Les migrations africaines", in Ailleurs, l'herbe est plus verte : histoire des migrations dans le monde, Collection Panoramiques, 1996.

- Riall Nolan, "Citadins bassari : la révolution tranquille", in Objets et Mondes, la Revue du Musée de l'Homme. Tome 21- Fasc. 4 - Hiver 1981.

- Abdou Salam Fall et Rokhaya Cissé, Migrations internationales et pauvreté en Afrique de l'Ouest, Ifan 2007. 